\title{
Optimized Design for Wireless Coil for Electric Vehicles Based on The Use of Magnetic Nano- Particles
}

\author{
Alberto Delgado \\ Polytechnic University of Madrid \\ (UPM) \\ Center of Industrial Electronic (CEI) \\ Madrid, Spain \\ a.delgado@upm.es \\ Jorge Rodriguez \\ Premo Group \\ Málaga, Spain \\ jorge.rodriguez@grupopremo.com
}

\author{
Jesús A. Oliver \\ Polytechnic University of Madrid \\ (UPM) \\ Center of Industrial Electronic (CEI) \\ Madrid, Spain \\ jesusangel.oliver@upm.es \\ Alejandro Jiménez \\ Premo Group \\ Málaga, Spain \\ alejandro.jimenez@grupopremo.com
}

\author{
José A. Cobos \\ Polytechnic University of Madrid \\ (UPM) \\ Center of Industrial Electronic (CEI) \\ Madrid, Spain \\ ja.cobos@upm.es
}

\begin{abstract}
Wireless Power Transfer has been endorsed as an innovative technology for charging electric vehicles. Since the autonomy of the electric vehicles is a key factor, the optimization of the wireless coil is a fundamental step in the design of these systems. The main inconveniences of these coils are the high weight and the fragility of ferrite. As a solution, some manufacturers are proposing coating the coil with polymers. New compound magnetic material based on magnetic ferromagnetic nanoparticles embedded in a polymeric material can offer significant mechanical advantages, withstanding mechanical vibrations, and concurrently improving the magnetic behaviour. In this paper, an optimization process by Finite Element Method will be performed, reducing the volume of ferrite and introducing the magnetic compound to keep the same magnetic features.
\end{abstract}

Keywords-electric vehicles, finite elements modelling, magnetic nanoparticles, wireless power transfer.

\section{INTRODUCTION}

The Wireless Power Transfer technology offers several benefits to electric vehicle e.g., extending vehicle autonomy and users charging comfort. Nevertheless, in order to achieve a significant improvement of vehicle autonomy, weight and size wireless coil optimization must be done [1]- [3].
For instance, the vehicle assembly of the standard SAE J2954 [4] for WPT1 and Z1 (the wireless coil that is in the car is called vehicle assembly in this standard) -and if the density of ferrite is about $5 \mathrm{~g} / \mathrm{cm} 3$ - could weight around $3 \mathrm{~kg}$ (magnetic core, winding and housing).

In this work, in order to solve the mechanical problems due to the fragility of the ferrite we proposed by Finite Element tools (Ansys Maxwell ${ }^{\circledR}$ ) an optimization process to reduce the volume of ferrite in a vehicle assembly. By doing so, the magnetic features of the final coil will be inferior. In order to simulate the wireless coil, the method explained in [5] is used.

To solve the problem of ferrite reduction, the use of compounds as the magnetic nanoparticles shows in [6] is proposed. These compounds can increase the energy of the wireless coils and reduce the losses in the shielding. In this paper, different combinations of designs with the magnetic composite are explained and compared.

This paper is structured into four sections. In Section II, a previous study of the state of the art related with the standard $\mathrm{SAE}$ is presented. Based on finite elements simulations, the features of the coils are presented. In Section III, a general strategy for the design of a receiver coil of an inductive power transfer system, where the emitter is defined, is presented. In TABLE 1

MAGNETIC PARAMETERS OF FE SIMULATIONS AT $85 \mathrm{KHz}$

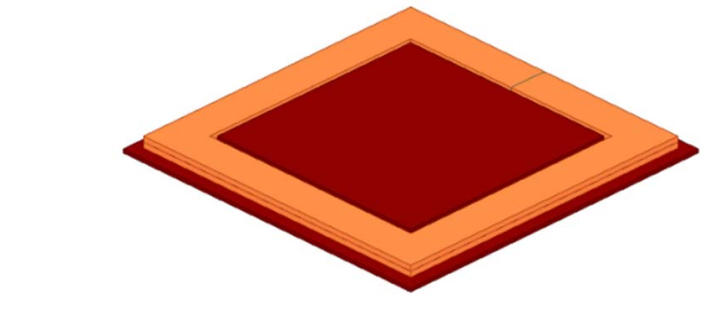

a)

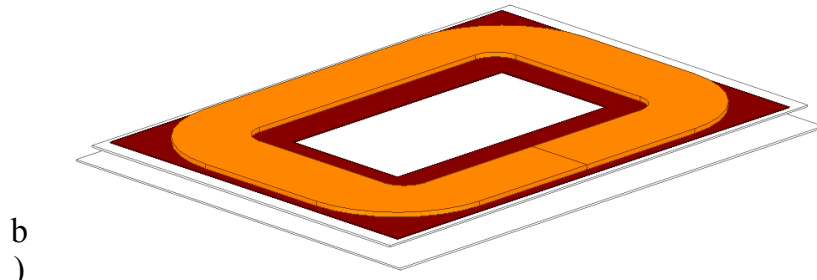

Figure 1. a) Vehicle assembly Appendix A of the standard SAE J2954 for WPT1 and Z1. b) Ground assembly Appendix G1 of the standard SAE J2954 for WPT1.

\begin{tabular}{llc}
\hline Variable & \multicolumn{1}{c}{ Description } & FE results \\
\hline$A_{L}$ & Inductance per turn of the vehicle assembly & $852 \mathrm{nH} / \mathrm{N}^{2}$ \\
& Quality factor of the vehicle assembly & 940 \\
$k_{0}$ & Coupling factor of the vehicle assembly with & $22.47 \%$ \\
& the ground assembly of the standard SAE & \\
$\eta_{0}$ & J295 at 100 mm & $99.60 \%$ \\
$w_{f}$ & Efficiency of the link with the ground & \\
& Weight of ferrite &
\end{tabular}

This work was supported in part by the Ministerio de Economia, Industria y Competitividad de España under Grants DPI2016-80953-R and RTC2016-4820-4 


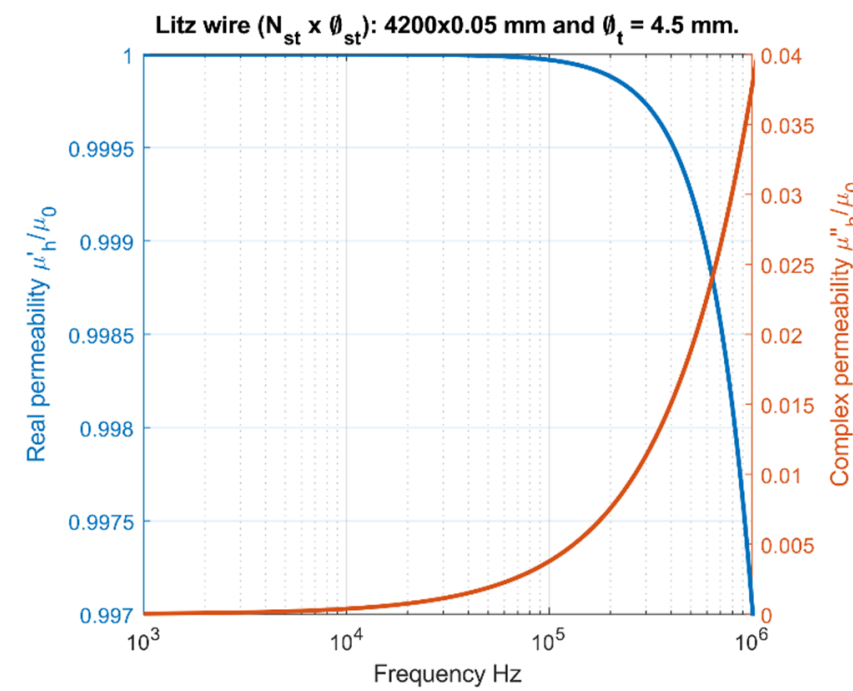

Figure 2. a) The coupling factor of a vehicle assembly vs the -Mid parameters. b) The inductance per turn of a vehicle assembly vs the -Mid parameters.

order to validate the simulations and the designs we measure the prototypes simulated by Finite Elements tools and these measurement results are presented in Section IV.

\section{PREVIOUS STUDY}

In this section, the magnetic features of a wireless coil system are described using Finite Element methods. The system is made with the vehicle assembly of a WPT1 and Z1 (Figure 1a) and the ground assembly of a WPT1 (Figure 1b) of the standard SAE J2954 [4]. The winding is construct using litz wire. This wire is made of 4200 strands where each strand has a diameter of $0.05 \mathrm{~mm}$.

The magnetic properties of this litz wire are shown in Figure 2 . The conductivity is constant $\left(3.06 \cdot 10^{7} \mathrm{~S} / \mathrm{m}\right)$ up to $400 \mathrm{kHz}$.

The magnetic properties of the design from SAE J2954 WPT1 and Z1 (Figure 1a) are in Table 1.

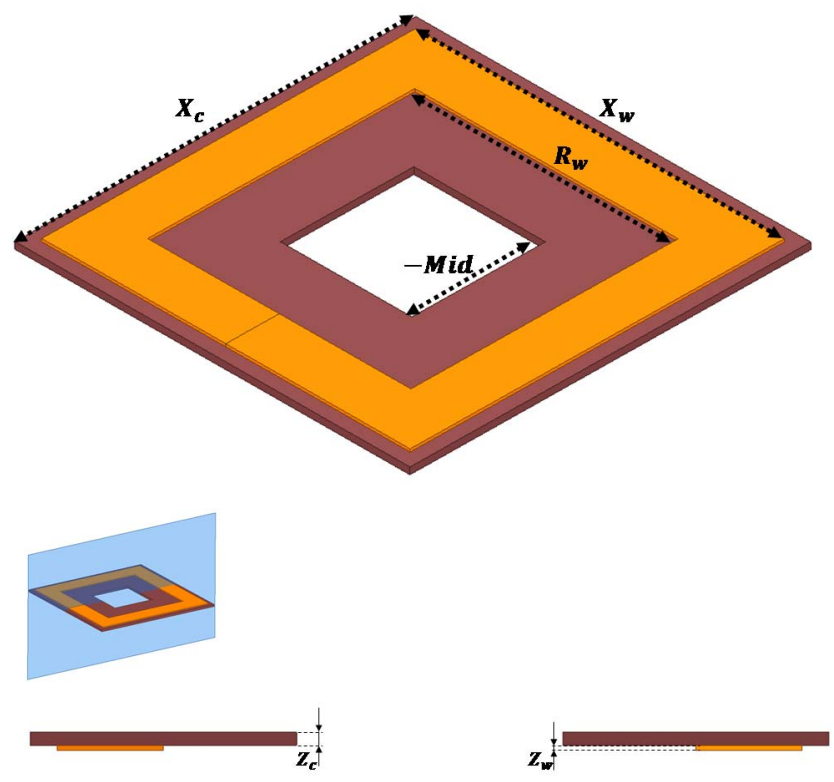

Figure 3. Detailed parameters description of a wireless coil on FE model in Ansys Maxwell.
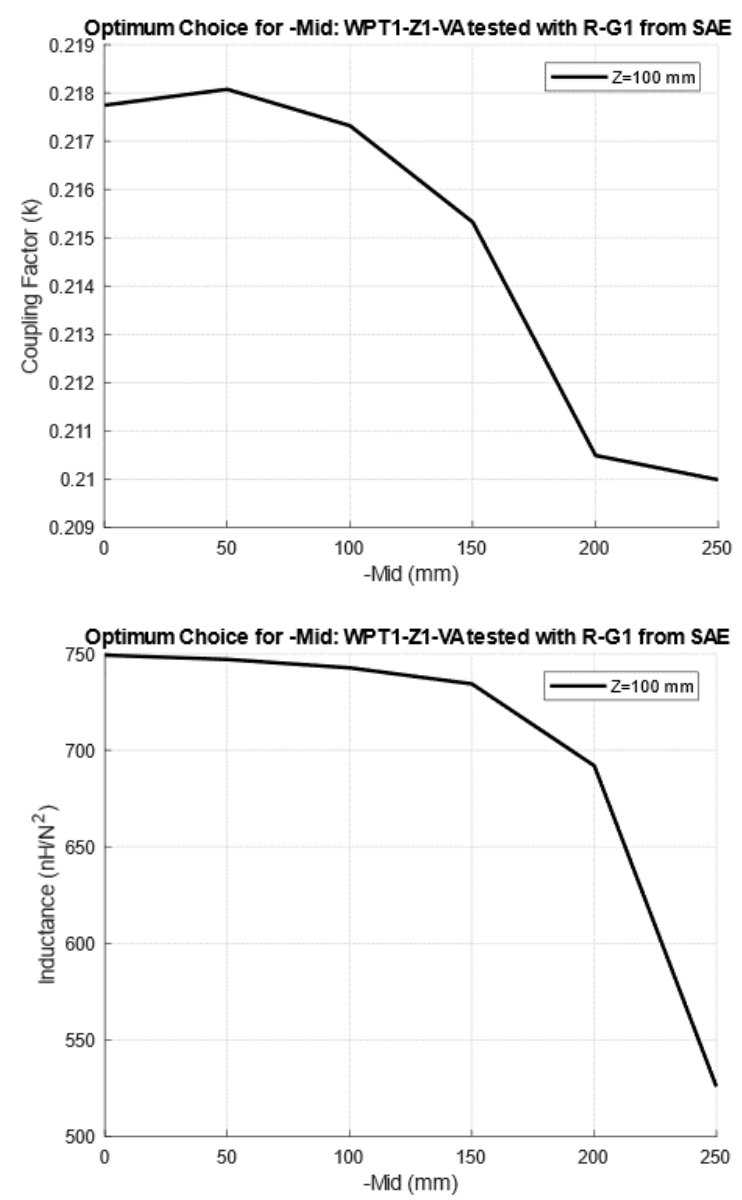

Figure 4. a) The coupling factor of a vehicle assembly vs the -Mid parameters. b) The inductance per turn of a vehicle assembly vs the -Mid parameters.

In this work, we are going to design a new structure for the vehicle (vehicle assembly). Then, the ground assembly from the standard SAE J2954 will be used for all simulations of the section III. In this way, we can compare the results obtained in this section with the results that we will obtain in the section III.

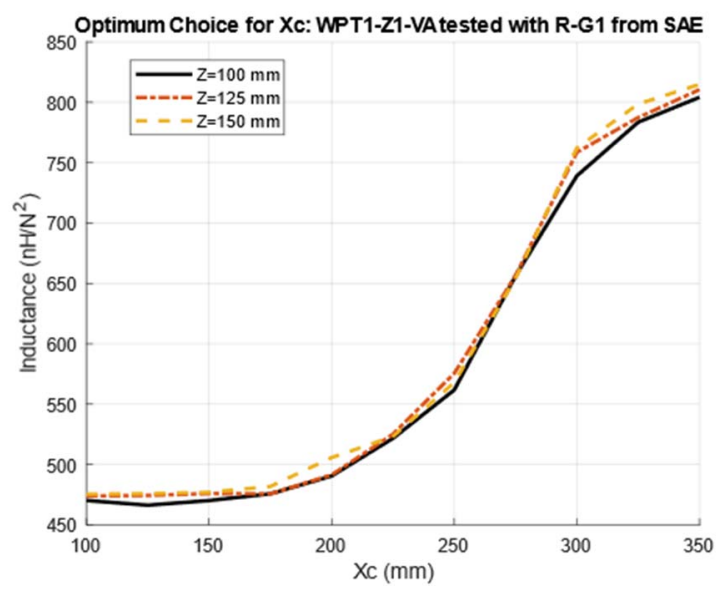

Figure 5. a) The inductance per turn of a vehicle assembly vs the Xc parameters. 

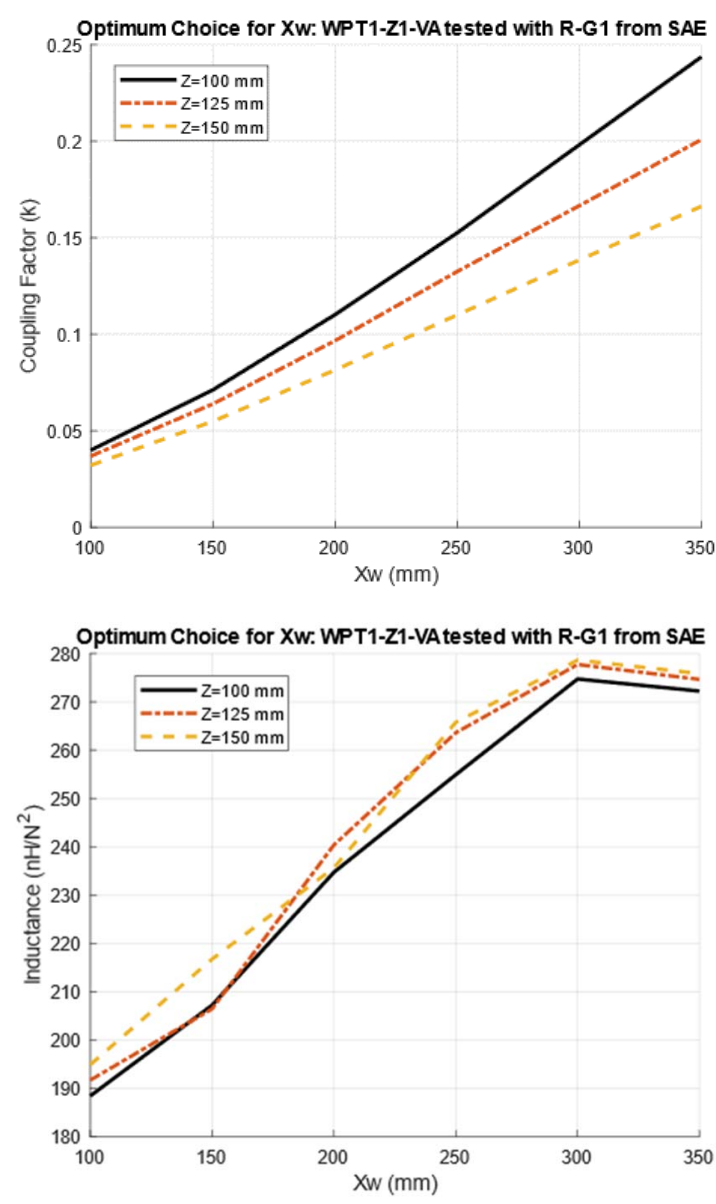

Figure 6. a) The coupling factor of a vehicle assembly vs the Xw parameters. b) The inductance per turn of a vehicle assembly vs the Xw parameters.

\section{Optimal Design of Vehicle ASSEMBly}

In order to optimize the vehicle assembly, we performed thousands of finite element simulations for a model of a wireless coil shows in Figure 3, where the geometry parameters Xw, Rw, Zw, Xc, Zc and -Mid, inter alia, are swept.

To perform these simulations, we define the maximum size of the coil at $300 \times 300 \times 15 \mathrm{~mm}$. Then, we configure all the parameters, and we vary only one at a time. In this way, we take the value that the characteristics of the coil improve.

Based on the results obtained, we choose the geometrical dimensions that allow to maintain the best features of the wireless coil and leaves to reduce the ferrite volume. For

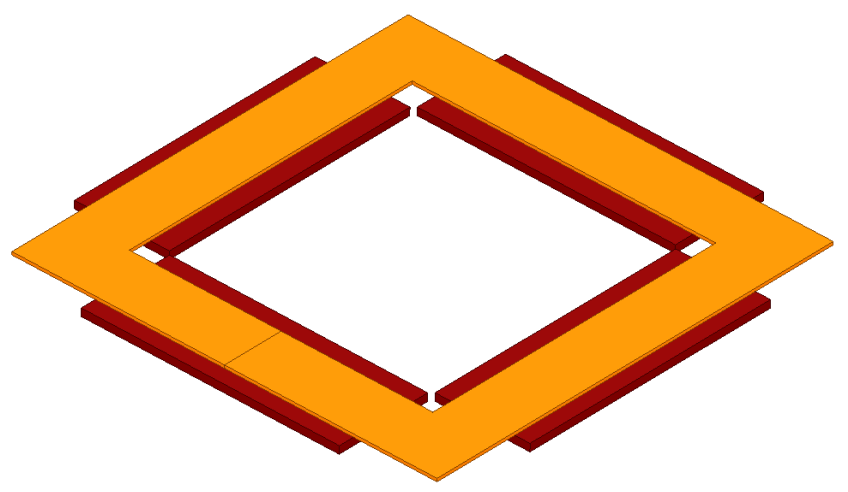

Figure 8. 3D view of the optimized wireless coil.
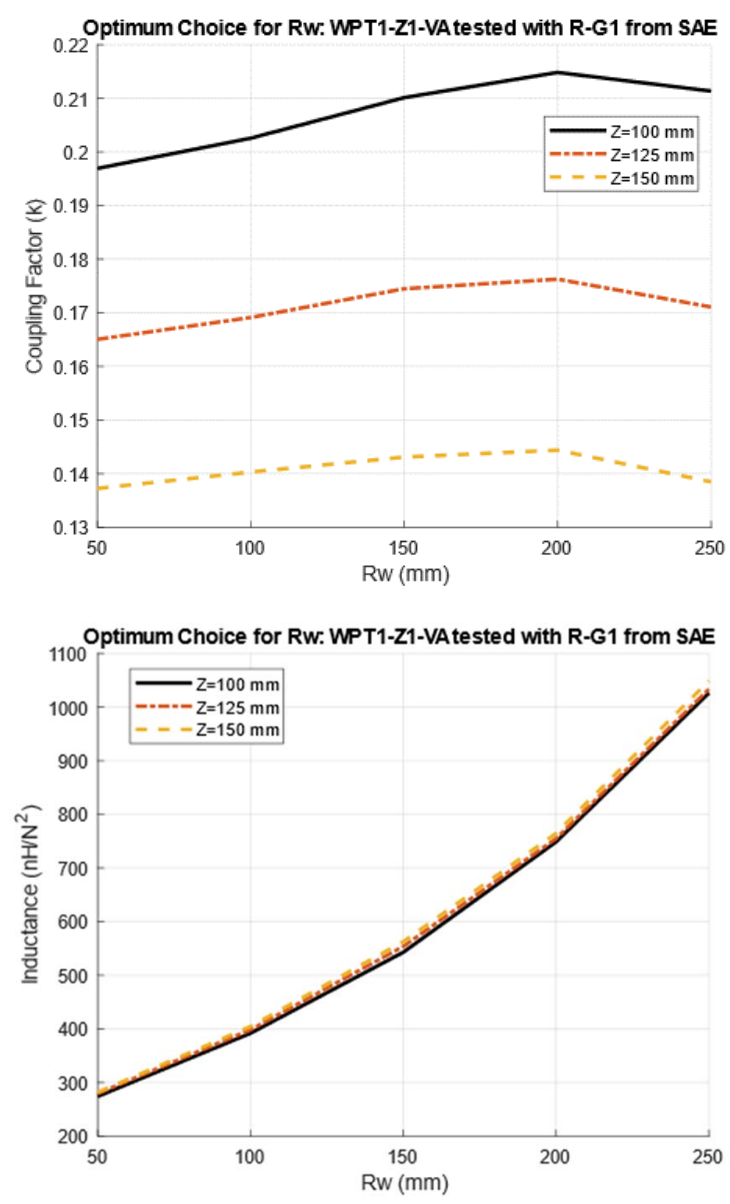

Figure 7. a) The coupling factor of a vehicle assembly vs the Rw parameters. b) The inductance per turn of a vehicle assembly vs the Rw parameters.

example, as we can see in Figure 4, if we remove the ferrite of the middle of the coil, we will keep the same inductance and the coupling factor. It means that we can save magnetic materials keeping the same magnetic properties.

Other thing is the importance of keeping ferrite under the winding. As we can see in Figure 5, where a coil is made using a $\mathrm{Rw}=200 \mathrm{~mm}$ and $\mathrm{Xw}=300 \mathrm{~mm}$, if we put the ferrite under the winding (from $X c=200$ up to $X c=300$ ), we will increase the inductance, then we can reduce the number of turns. It means that we will save copper material and then we can reduce the weight.

Concerning the winding, if we perform a simulation where the magnetic core $\mathrm{Xc}$ is $300 \mathrm{~mm}$ :

TABLE 2

GEOMETRY PARAMETERS OF THE OPTIMIZED DESIGN

\begin{tabular}{cc}
\hline$X w$ & $280 \mathrm{~mm}$ \\
\hline$R w$ & $200 \mathrm{~mm}$ \\
\hline$Z w$ & $2-3 \mathrm{~mm}$ \\
\hline$X c$ & $300 \mathrm{~mm}$ \\
\hline$Z c$ & $3 \mathrm{~mm}$ \\
\hline- Mid & $175 \mathrm{~mm}$
\end{tabular}


TABLE 3

MAGNETIC PARAMETERS OF FE SIMULATIONS AT $85 \mathrm{KHz}$

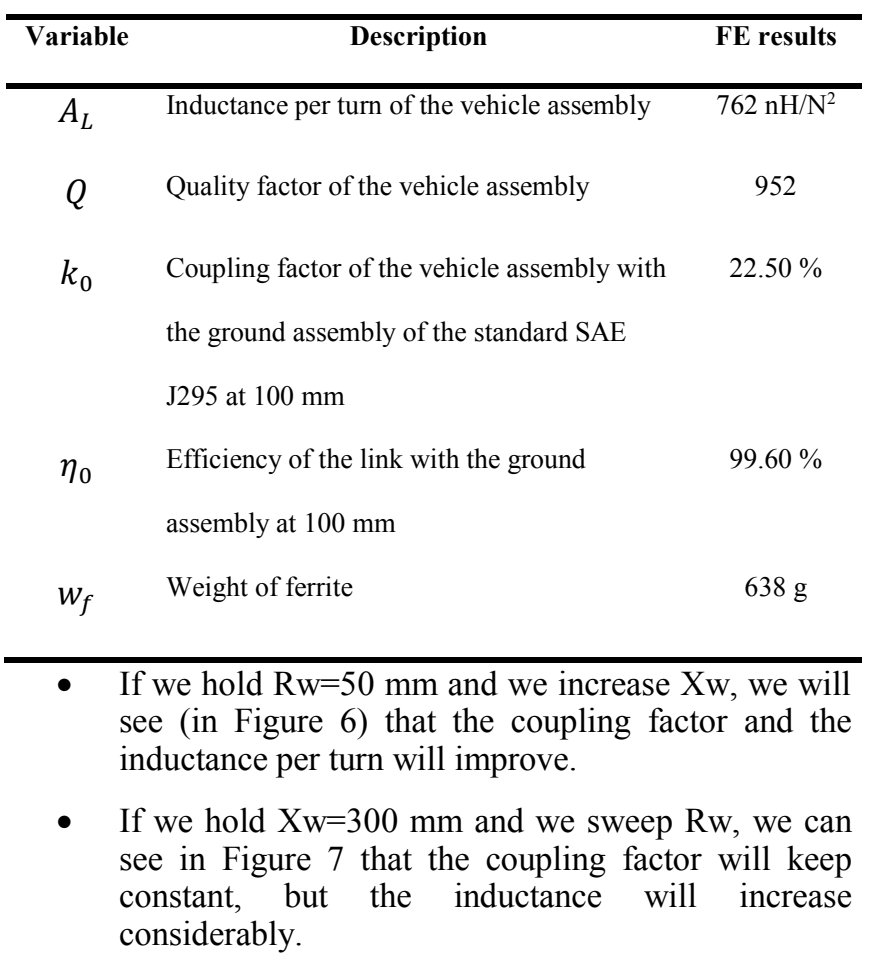

Based on these results, the optimal design is shown in Figure 8 and the geometrical parameters are in Table 2.

In this design, we remove the ferrite of the corners to improve the mechanical features. As a result of the implementation of this decision, the inductance will decrease, but the coupling factor will keep the same.

The magnetic properties of the optimal design of Figure 8 from Finite Element simulations are in Table 3.

By reducing the volume of ferrite, the magnetic properties of the wireless coil will be worse than those of the standard SAE J2954 vehicle assembly. By doing that, the inductance will decrease, the radiation will increase and a consequence of that, the losses on the shielding will increase.

Therefore, the use of magnetic nanoparticles -embedded in the polymer that covers the coil- can improve the magnetic properties of the wireless coil so that it can achieve

TABLE 4

MAGNETIC PARAMETERS OF FE SIMULATIONS AT $85 \mathrm{KHz}$

\begin{tabular}{clcc}
\hline Variable & \multicolumn{1}{c}{ Description } & Figure 9a & Figure 9b \\
\hline$A_{L}$ & Inductance per turn of the & $819 \mathrm{nH} / \mathrm{N}^{2}$ & $932 \mathrm{nH} / \mathrm{N}^{2}$ \\
& vehicle assembly & & \\
& & 1021 & 1164 \\
$k_{0}$ & Quality factor of the & & \\
& Coupling factor of the & $22.80 \%$ & $21.65 \%$ \\
& new vehicle assembly & & \\
$\eta_{0}$ & Efficiency of the link & $99.62 \%$ & $99.62 \%$ \\
\end{tabular}
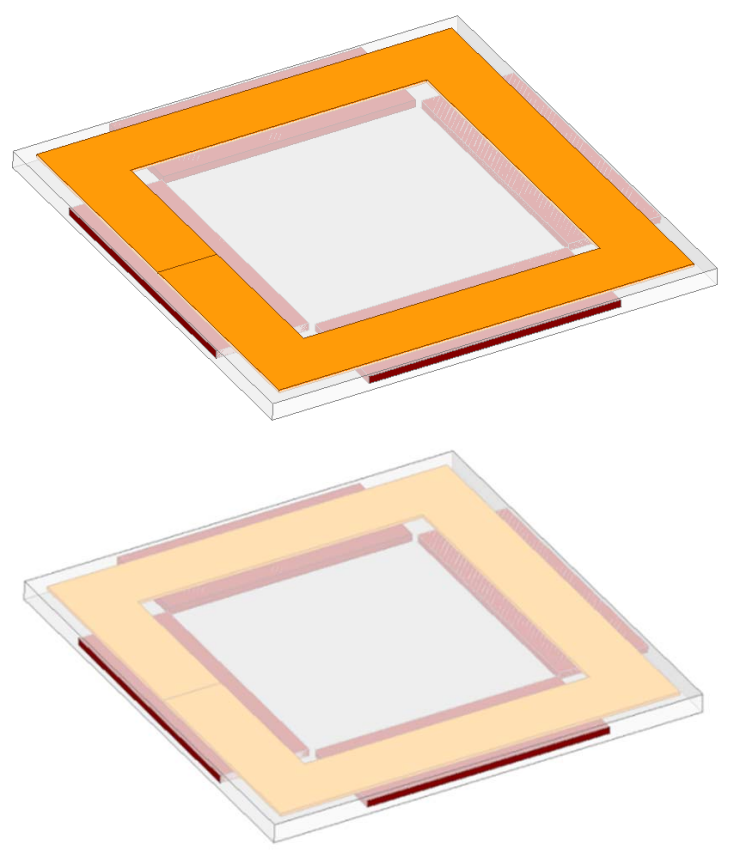

Figure 9. Illustration of the vehicle assembly with the magnetic nanoparticles compound

magnetic properties like the coil of the standard SAE J2954.

Thus, different models have been simulated: the first model is with the compound covering everything but the winding (see Figure 9a) and the second model with the whole wireless coil covered (see Figure Error! Reference source not found.9b).

In the first case (Figure 9a), we use the compound as a magnetic core. By doing so, we can reduce the fringing flux because the magnetic field has in the compound a path with less reluctance than the air. In this way, we can guide the magnetic field to the ferrite core.

In the second case (Figure 9b), also we use the compound to cover the winding. By doing so, we can reduce the leakage flux keeping this energy in the compound. Therefore, the inductance will increase. Also, the coupling factor will decrease.

The magnetic compound is made of polymer FMS0.250 and the ferrite is $3 \mathrm{C} 94$. The percentage by weight of ferrite is

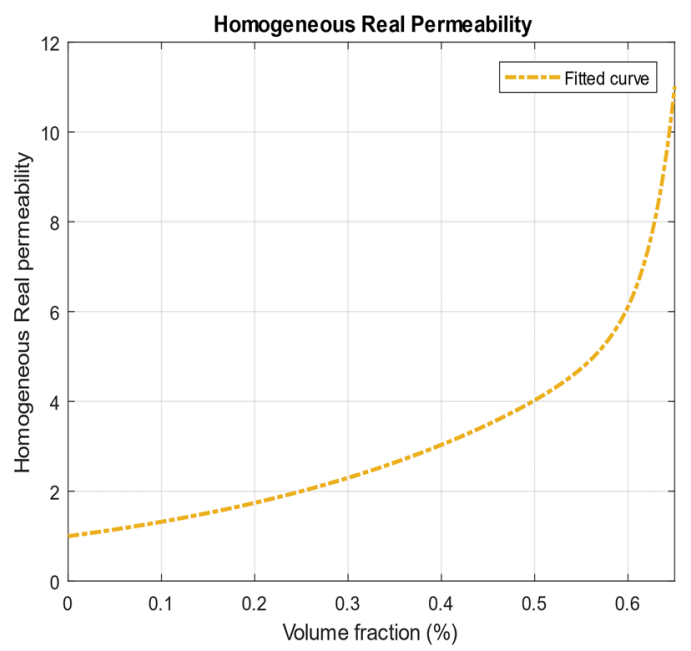

Figure 10. Homogeneous real permeability of the compound depending on the volume fraction 


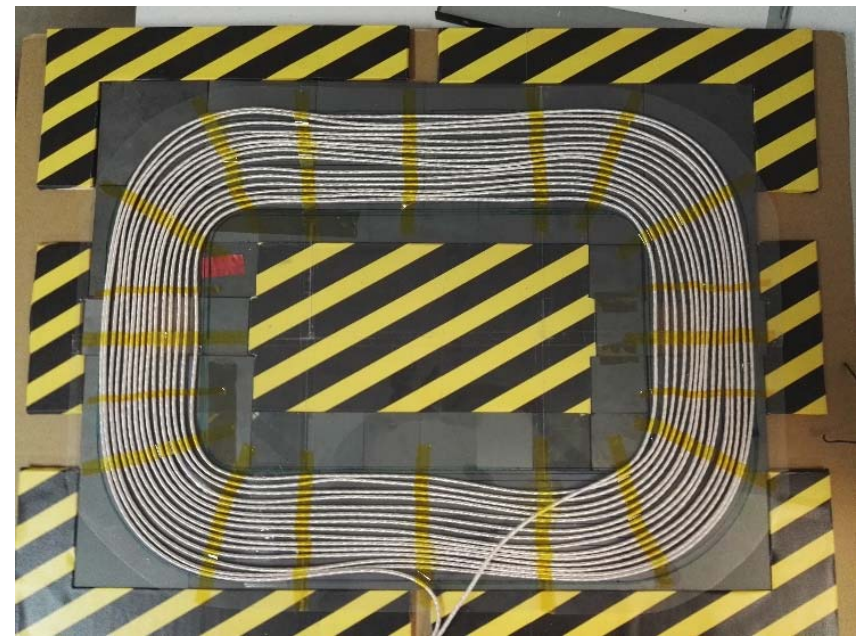

Figure 11. Prototype of the Ground Assembly from the standard SAE J2954

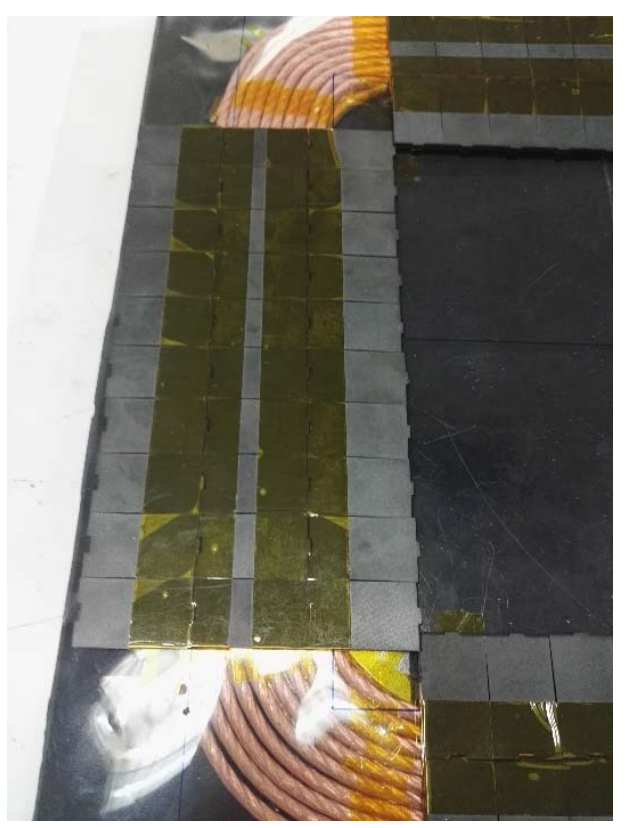

Figure 12. Illustration of the magnetic core made of links of ferrite of the optimum vehicle assembly.

$65 \%$. The density of this polymer is around $3 \mathrm{~g} / \mathrm{cm}^{3}$ and the density of the ferrite is around $5 \mathrm{~g} / \mathrm{cm}^{3}$, the volume fraction of ferrite is around $52 \%$.

According to [6], as we can see in Figure 10 this percentage means that we have approximately a permeability of 4 .

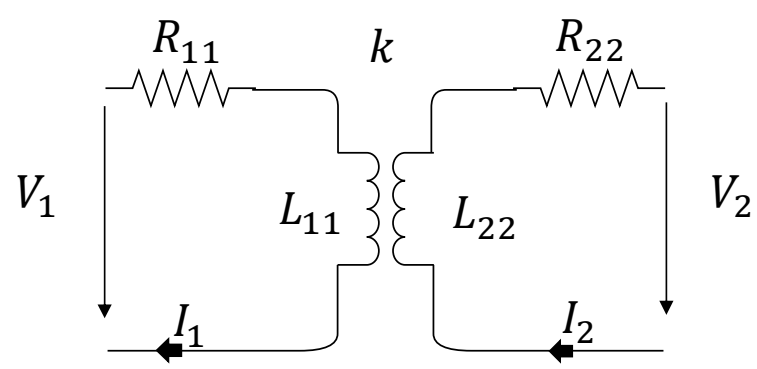

Figure 13. Illustration of the schematic of a wireless link.

Even if the permeability of the compound is low, in terms of reluctance, the compound has 4 times less reluctance than the air.

The magnetic properties of the optimal design (Figure 9) with the compound are in Table 4.

\section{EXPERIMENTAL VALIDATION}

Based on the previous section, the optimal prototypes of figures $8,9 \mathrm{a}, 9 \mathrm{~b}$ and the ground assembly of the standard SAE J2954 have been built.

The magnetic core of the ground assembly is made with tiles of ferrite 3C95 from Ferroxcube (Figure 11).

The winding of the ground assembly is made with a litz wire of 630 strands of $0.1 \mathrm{~mm}$ of diameter from Elektrisola.

The magnetic core of the vehicle assembly but the compound is made with links of ferrite (Figure 12) that can improve the mechanicals characteristic.

The winding of the optimum vehicle assembly is made with a litz wire of 1125 strands of $0.07 \mathrm{~mm}$ of diameter Elektrisola.

In order to measure the magnetic properties of the wireless link system, Bode100 (from Omicron $(\circledR)$ ) is used. Then, if we have a wireless link as Figure 13, we can measure the primary inductance as:

$$
\left.\frac{V_{1}}{I_{1}}\right|_{I_{2}=0}=Z_{11}=R_{11}+j \omega \mathrm{L}_{11}
$$

The secondary inductance as:

$$
\left.\frac{V_{2}}{I_{2}}\right|_{I_{1}=0}=Z_{22}=R_{22}+j \omega \mathrm{L}_{22}
$$

And the mutual inductance as:

$$
\left.\frac{V_{1}}{I_{2}}\right|_{I_{1}=0}=Z_{12}=R_{12}+j \omega \mathrm{L}_{12}
$$

TABLE 5

MEASUREMENT OF THE MAGNETIC PARAMETERS AT $85 \mathrm{KHz}$

\begin{tabular}{ccccc}
\hline Variable & Ground Assembly & Figure 8 & Figure 9a & Figure 9b \\
\hline $\mathrm{A}_{\mathrm{L}}$ & $1200 \mathrm{nH} / \mathrm{N}^{2}$ & $785 \mathrm{nH} / \mathrm{N}^{2}$ & $806 \mathrm{nH} / \mathrm{N}^{2}$ & $968 \mathrm{nH} / \mathrm{N}^{2}$ \\
$\mathrm{Q}$ & 373.85 & 606.17 & 622.20 & 747.70 \\
$\mathrm{k}_{0}$ & - & $27.6 \%$ & $27.6 \%$ & $25.2 \%$ \\
$\eta_{0}$ & - & $98.48 \%$ & $98.50 \%$ & $98.50 \%$ \\
\hline
\end{tabular}


Then, the coupling factor is:

$$
k=\frac{L_{12}}{\sqrt{L_{11} L_{22}}}
$$

Where the efficiency of the link can be calculated as:

$$
\eta=1-\frac{2}{Q_{i} L_{i}}
$$

Where $\mathrm{Q}$ is the quality factor that can be expressed as:

$$
Q_{i}=\frac{\omega L_{i}}{R_{i}}
$$

Each optimum vehicle assembly has been measured with the ground assembly. The results are shown in Table 5 .

\section{CONCLUSION}

Summing up, in this paper, we have presented assorted designs of wireless coils of a Wireless Power Transfer system for electric vehicles reducing the volume of ferrite including magnetic nanoparticles in the polymer that will protect the wireless coil. To optimize the designs a set of Finite Element simulations have been performed. To test the FE results, various experimental prototypes -including the polymer with the magnetic nanoparticles- and the ground assembly were built. The optimized designs were compared with the vehicle assembly of the standard SAE J2954. The designs with magnetic nanoparticles -even having less ferrite- present characteristics similar to the wireless coil made with a compact ferrite plate as a magnetic core. The weight of the optimized coil is $1.5 \mathrm{~kg}$ (including the winding), when the standard SAE J2954 presents a coil which weights $2 \mathrm{~kg}$.

\section{ACKNOWLEDGMENT}

We would like to thank Ferroxcube for their support by providing the magnetic materials to construct the cores and Elektrisola for their support supplying the litz wire to build the windings.

\section{REFERENCES}

[1] Bosshard, R., Kolar, J. W., Mühlethaler, J., Stevanović, I., Wunsch, B., \& Canales, F. (2015). Modeling and \$leta \$-\$lalpha \$-Pareto Optimization of Inductive Power Transfer Coils for Electric Vehicles. IEEE Journal of Emerging and Selected Topics in Power Electronics, 3(1), 50-64

[2] Shin, J., Shin, S., Kim, Y., Ahn, S., Lee, S., Jung, G., ... \& Cho, D. H. (2014). Design and implementation of shaped magnetic-resonancebased wireless power transfer system for roadway-powered moving electric vehicles. IEEE Transactions on Industrial electronics, 61(3), 1179-1192.

[3] Li, S., \& Mi, C. C. (2015). Wireless power transfer for electric

[4] vehicle applications. IEEE journal of emerging and selected topics in power electronics, 3(1), 4-17.

[5] J. Schneider, "SAE J2954 overview and path forward. SAE International." 2013.

[6] A. Delgado, G. Salinas, J.A. Oliver, J.A. Cobos and J. Rodriguez, "Equivalent Parameters of Round and Litz Wire Conductors to Obtain an Equivalent Layer to Accelerate Finite Element Simulations of Wireless Power Transfer System," in 2018 IEEE Energy Conversion Congress and Exposition (ECCE), 2018.

[6] A. Delgado, G. Salinas, J.A. Oliver, J.A. Cobos and J. Rodriguez, "Finite Element Modelling of Litz wire Conductors and Compound Magnetic Materials based on Magnetic Nano-particles by means of Equivalent Homogeneous Materials for Wireless Power Transfer System," in 2018 IEEE Workshop on Control and Modeling for Power Electronics (COMPEL), 2018. 